\title{
Factors influencing fertility preferences of currently married men in Kenya
}

\author{
Janet Naisoi Mashara \\ Department of Humanities, Karatina University, \\ P.O. Box 1637-00200, NAIROBI. \\ naisoimashara@gmail.com
}

\begin{abstract}
Fertility preferences are central in determining the future fertility of the society particularly where and when those desires are implemented. The socio-cultural structures in most African communities have given men the mandate to decide in all aspects of life including family sizes and fertility behaviors. Information on fertility preference in Kenya especially regarding men is very scanty. This study therefore specifically sought to establish the effects of sociodemographic; socio-economic; and socio-cultural factors on fertility preference of currently married men in Kenya. Data was drawn from sample size of 1,757 married men aged 15-54 years who were asked questions on various topics including fertility preference during the $2008 / 9$ KDHS. The study findings revealed that age, number of living children, education, region, occupation, type of marriage and number of living sons were significant factors associated with the desire for additional children at $0.001,0.01$ and 0.05 significance level. In conclusion, fertility preference of currently married men in Kenya is influenced mainly by demographic (age \& Number of living children); socio-economic (education \& region) and socio-cultural factors (type of marriage \& Number of living sons). Recommendations: i) Education for men should be emphasized because education was discovered to have a significant negative effect on the fertility preference; ii) Policies that aim at integrating population into development should be encouraged so as to foster socio-economic development in all the regions and hence minimize the regional disparities as it relates to fertility preferences; iii) Further studies, both qualitative and quantitative, to be carried out in order to explore the socio-cultural religious beliefs, norms and attitudes of men in regards to the value of children; v) Qualitative studies needs to be conducted in the North Eastern region to find out the driving forces for glaringly high fertility preference other than low literacy level.
\end{abstract}

Key Words: Fertility preference, Desire for Additional Children, Sex preference, Education, age.

\section{Introduction}

In recent decades fertility has declined at a rapid pace in a majority of developing countries including Kenya. Kenya's population size has grown from 5.4 million in 1948 to 38.6 million in 2009 with the total fertility rate ranging between 4.9 in 2003 and 4.6 in 2008/9 revealing a stall in fertility. Studies have shown that fertility preferences are potentially important in shaping the fertility of the society as future fertility behavior will most likely be affected by the currently observed fertility preferences (Ayehu, 1998). Although reproduction related goals and decisions are done by a couple, studies and surveys on fertility indicate that fertility preferences differ among husbands and wives with husbands' preference being higher than that of their wives. This could be linked to the position that they command in terms of decision making as the heads of households. In explanations of fertility extremes, or of the discrepancies between desired and actual fertility, the effect of partners' holding different preferences have tended to be overlooked (Adamchak and Adebayo, 1985; Bankole and Singh, 1998).

Understanding the fertility preference of married men in Kenya is of paramount importance given the effect it has on future fertility when the preferences are implemented. It is also important to family planning programmes because it helps determine the need for contraception, whether for spacing or limiting births, and the extent of unwanted and mistimed pregnancies.

The main objective of this study is to determine the factors that contribute to fertility 
preference of men in Kenya. The specific objectives of this study are: i) To establish the effect of socio-demographic factors on fertility preferences among currently married men in Kenya; ii)To examine the effect of socioeconomic factors on fertility preference of currently married men in Kenya; iii) To investigate the effect of socio-cultural factors on fertility preference of currently married men in Kenya.

The paper has divided into 11 sections that is: abstract; introduction; problem statement; data sources and methodology; literature review; conceptual framework; operationalization of variables; Findings and discussion; conclusion; recommendation and references.

\section{Problem Statement}

Studies that have been done on fertility preference show that fertility preference measures are consistent have predictive validity and have useful meaning. Personal preference is seen as an important factor to shaping individual's behavior. Further, it is noted that individuals' fertility preference is a medium through which the future fertility preference is shaped. Longitudinal studies have also shown that individual fertility preferences are often sufficient enough to have a large effect on subsequent fertility (Foreit and Suh, 1980).

In Kenya however, studies on fertility measures including that of fertility preference have focused mainly on women. Fertility desires expressed by the male counterparts remains largely unknown. Research on how men form opinions about family size, fertility and family planning and how they can be influenced is required since the women's view may not reflect that of men. Given that men are the dominant decision-makers in the African families, it is important that their preference and reproductive behaviours are explored.

This could help in explaining the causes of fertility transition and fertility stalling as observed in Kenya. In addition, it will be essential in setting priorities to allocate the scarce resources towards the factors that could greatly influence fertility desires of currently married men in Kenya taking into consideration the ongoing Reproductive Health Programmes that advocate for male involvement. This study intended therefore to look at the demographic, sociocultural, and socio-economic factors that influence fertility preference of married men in Kenya.

\section{Data sources and methodology}

The data used for this study is drawn from the 2008-9 Kenya Demographic and Health Survey (KDHS). The KDHS is a nationally representative sample survey of 8,444 women age 15 to 49 and 3,465 men age 15 to 54 selected from 400 sample points (clusters) throughout Kenya. The survey was householdbased and therefore a sample of 10,000 households was drawn from the population residing in the households in the country. This sample was constructed to allow for separate estimates for key indicators for each of the eight provinces in Kenya, as well as for urban and rural areas separately. Compared with the other provinces, fewer households and clusters were surveyed in North Eastern province because of its sparse population. A deliberate attempt was made to oversample urban areas to get enough cases for analysis. As a result of these differing sample proportions, the KDHS sample is not self-weighting at the national level; consequently, all tables except those concerning response rates are based on weighted data. KNBS and ICF Macro. (2010)

From the overall sample of 3,465 men, the study focused on data collected from the men's questionnaire, which yielded a sample of a sample of 1,757 married men aged between 1554 who were interviewed on various topics including fertility preference.

\section{Literature Review}

Economic fertility theories have equated children children like household goods. That children in the modern society are viewed as liabilities and they compete with the goods such that parents are forced to make rational decisions whether to have an additional child or not based on their economic situations at that moment (Becker 1960; Caldwell, 1976; Mahmud and Ringheim, 1997). The key factors that act in control of fertility related decisions on family size include: education; cost of fertility regulation; potential output of children and demand for children (Easterline, 1973; Singh and Casterline, 1985). Formal education heightens a person's consciousness on the real and perceived costs and utilities of children (DeRose et al. 2002). Caldwell's (1982) wealth flow theory also emphasizes the value of large families in less developed countries.

Fertility preference varies with age. For instance the difference is more pronounced in Africa where the mean ideal family size ranges from 8.8 in the 20-29 years age group of men to 16.6 for men aged 60 years and over (Ezeh et al., 1996). The positive relationship between family size preference and age can be 
interpreted in two ways. First, it may point to changing reproductive norms where younger men, who are likely to be more educated, may be motivated easily and therefore act as forerunners of the new reproductive norm, while the preference of older men may reflect traditional reproductive norms. On the other hand, older men may adjust their fertility desires upward over the life course to accommodate their own growing number of children (Ezeh et al., 1996). Various other studies have demonstrated that the association between additional children and actual fertility is negative. That the more the number of living children a couple has the lesser the likelihood that they woud desire an additional child (Sirageldin 1977; Muganzi and Takona, (1994; Bulatao and Fawcet, 1983). However other case studies (Lighbourne and MacDonald 1982) have revealed that the average number of children desired tends to increase quite noticeably with almost every increase in the number of children living.

Urban people prefer smaller families. Family size preference also varies with regional variations of place of residence (Knodel et al., 1996; Singh and Casterline 1985; Mahmud and Ringheim, 1997; Ali, 2000; Ekisa and Hinde 2006). Studies have also showed that the association between desire for additional children and the actual family size is inverse, that is; as family building process progresses further, parents tend to stop childbearing. In addition for an individual to desire an additional child, several factors have to be considered other that the number of living children that one already has. Generally, factors that have been associated with these personal preferences include socio-demographic (age and number of living children), socio-cultural (type of marriage and sex preference), and socio-economic (education, region, place of residence, occupation and wealth) factors for which this study seeks to examine. Studies have revealed a significant relationship between region and desire for more children. Kimondo, 2003; Mutetei, 1998; Macro International Inc., 2010 affirmed that fertility preference varies by region.

The desire to have or not to have children depends not only on the age and actual family size but also on the sex composition of the children a person has already produced. As presented in various studies, there is preference for sons in most of the sub-Saharan African countries for economic and cultural reasons (Dahl and Moretti, 2008). With respect to type of marriage, polygynously married men report higher fertility desires than monogamously married men (Ezeh et al., 1996; Ezeh 1993).

\section{Conceptual Framework}

This study is conducted within the economic utility theories of fertility. The economic theory of fertility postulates that children are seen as similar to commodities and other goods. The number of children who will be brought forth depend on the relative preference for children compared to other goods and the relative cost of children verses other goods. This theory is largely a demand-oriented theory based on individual circumstances facing each threshold. Its authors argue that fertility decisions are based on the current assessment of costs and benefits of the next birth and that decision is made on one birth at a time. After each birth, a couple make a decision to have another birth, postpone another birth or stop reproduction, based on the constantly changing payoff structure which is sensitive to changes in the economic and social circumstances (Udry, 1983; Becker, 1960 and Willis, 1973).

The analysis employed Pullum (1980) conceptual framework. In his Model, Pullum proposes that every woman at every time has an entire preference function, which describes the relative utility of each family size that she could possibly have (in an abstract sense). That function together with her current family size will generate her statements about desire for additional children. If she attaches higher utility to a greater family size than the one she currently has then she will state a preference for more children. Her response to question on ideal family size will be the model value of the function. Pullum's model represents the relationship over time between a) the underlying preference, b) the actual family size, c) the related controls, and d) the pattern of contraceptive use.

The theoretical statement postulated by Pullum shows the existence of a relationship between fertility preference and other demographic, socio-cultural and socio-economic variables. The framework modified in this study to establish the factors influencing the fertility preference of currently married men in Kenya.

\section{Operationalization of the Variables}

The dependent variable for this study was men's fertility preference. Independent variables included: socio-demographic, socio-cultural and socio-economic factors. The operational definitions of the variables are as explained below. 


\section{Dependent Variable}

Fertility preference was measured by the "Desire for additional children". In the 2008-09 KDHS men questionnaire, the questions were framed differently depending on whether one's wife was pregnant or not. Questions included "Would you like to have (a/another) child, or would you prefer not to have any (more) children?" to the respondent whose wife/partner is not pregnant, and "After the child(ren) you and your (wife(wives)/partner(s)) are expecting now, would you like to have another child, or would you prefer not to have any more children?" to the respondent whose wife (wives)/partner(s) is pregnant.

The responses given were all alphanumeric and were later dichotomized so that all responses indicating "desire for more children irrespective of the timing were recorded as 1 , otherwise 0 .

\section{Independent Variables}

The independent variables considered for this study include:

Type of Place of Residence: (urban and rural) Region: (Central, Coast, Eastern, Nyanza, Riftvalley, Western North-Eastern Nairobi)

Number of living Children: (none, 1-2, 3-4 and 5 or more children).

Education: (No education, Primary education, Secondary education, Higher education)

Age: (15-24, 25-34, 35-44, 45-54).

Number of Living Sons: (none, 1-2, 3-4 and 5 or more sons).

Wealth Index: Wealth index is based on household ownership of material possessions such as radio, television, telephone, refrigerator, bicycle, motorbike, and car. It also includes the housing quality, whether the house has electricity, a finished floor, and a permanent roof. The assumption is that men in the higher wealth index are expected to have lower fertility preference.

Occupation: The responses were categorized into 10 groups at the time of interview as no work, do not know, sales, household \& domestic, services, skilled manual workers, unskilled manual workers, professionals (doctor, technical personnel and managers), agriculture self employed and agriculture workers. The respondents were grouped into 2 categories as $0=$ agriculture and $1=$ others. Agriculture group includes agriculture self-employees and agriculture workers. Other group includes all the rest but excludes the group who answered "do not know". The latter group is excluded from the total number as missing values.

Type of Marriage: he is (monogamous or polygamous).

Factors Associated with the Desire for Additional Children

This section presents tabulated findings on the basic characteristics of the study population; the bivariate and multivariate analysis and subsequent discussions of the same.

Table 7.11 below shows the percentage distribution of the study population in relation to the study variables.

Table 7.11: Percentage Distribution of the study population according to the study Variables

\begin{tabular}{|l|l|l|}
\hline Variables & Percent & $\mathbf{N}=\mathbf{1 , 7 5 7}$ \\
\hline Desire for additional children & & \\
Desires & 56.29 & 989 \\
Does not Desire & 43.71 & 768 \\
\hline Respondent's age & 6.66 & \\
$15-24$ & 38.02 & 668 \\
$25-34$ & 31.82 & 559 \\
$35-44$ & 23.51 & 413 \\
$45-54$ & & \\
No. of Living Children & 6.26 & 110 \\
0 & 33.30 & 585 \\
$1-2$ & 31.47 & 553 \\
$3-4$ & 28.97 & 509 \\
\hline $5+$ & & \\
\hline Highest Education Level & 9.45 & 166 \\
No Education & 48.15 & 846
\end{tabular}




\begin{tabular}{|l|l|l|}
\hline Secondary + & 42.40 & 745 \\
\hline Region & 13.38 & 235 \\
Nairobi & 9.73 & 171 \\
Central & 14.74 & 259 \\
Coast & 11.84 & 208 \\
Eastern & 15.48 & 272 \\
Nyanza & 16.68 & 293 \\
Rift Valley & 11.16 & 196 \\
Western & 7.00 & 123 \\
North Eastern & & \\
\hline Wealth Index & 32.67 & 574 \\
Low & 14.23 & 250 \\
Medium & 53.10 & 933 \\
\hline High & & \\
\hline Type of Place of Residence & 35.46 & 623 \\
Urban & 64.54 & 1134 \\
Rural & 32.67 & \\
\hline Occupation & 67.33 & 574 \\
Agriculture & & 1183 \\
\hline Other & 91.52 & \\
\hline Type of marriage & 8.48 & 1608 \\
Monogamous & & 149 \\
\hline Polygamous & 20.49 & 360 \\
\hline No. of Living Sons & 53.73 & 944 \\
0 & 18.95 & 333 \\
1-2 & 6.83 & 120 \\
\hline 3-4 & & \\
\hline 5+ & & \\
\hline
\end{tabular}

\section{Discussion of the basic characteristics of the study population}

According to the results, majority of married men (56\%) desire additional children. with respect to age, about 7 percent of married men are below the age of 25 years; majority of the Majority of the respondents (70\%) are aged between 25 and 44 years and about 28 percent are aged between $45-54$ years. There is a small difference between the percentage of married men with 12, 3-4 or 5+ living children as represented by 33 , 32 and 29 percent respectively. About 6 percent of them have no living children. Regionally, the distribution of the study population ranges between about 10 percent in Central and 17 percent in Rift Valley with the lowest percentage (7 percent) living in North eastern. Regarding type of place of residence, a majority of men $(65 \%)$ reside in the rural areas.

With regards to educational attainment, about 10 percent of married men have no education; almost half (48 percent) of them have primary education, 42 percent have secondary + education.

The results also illustrate that the distribution of married men by wealth index varies with the majority percentage (53 percent) being in the high wealth index.

In relation to occupation about 33 percent of married men work in the agriculture sector while 67 percent work outside agriculture. The percentage distribution of respondents by type of marriage shows that about 92 percent of married men in Kenya are monogamous type of marriage. A majority of married men in Kenya (54\%) have 1-2 living sons.

\section{Differentials in the Desire for Additional Children}

This section presents the results of the association between the outcome (Desire for Additional children) and explanatory variables (demographic, socio-economic and sociocultural). The results showing the percentage distribution of currently married men's desire for additional children by their demographic, socioeconomic and socio-cultural characteristics together with the chi-square test statistics are as illustrated in table 7.21. 
Table 7.21 Percentage distribution of the Study Population according to the Desire for Additional Children and the selected variables

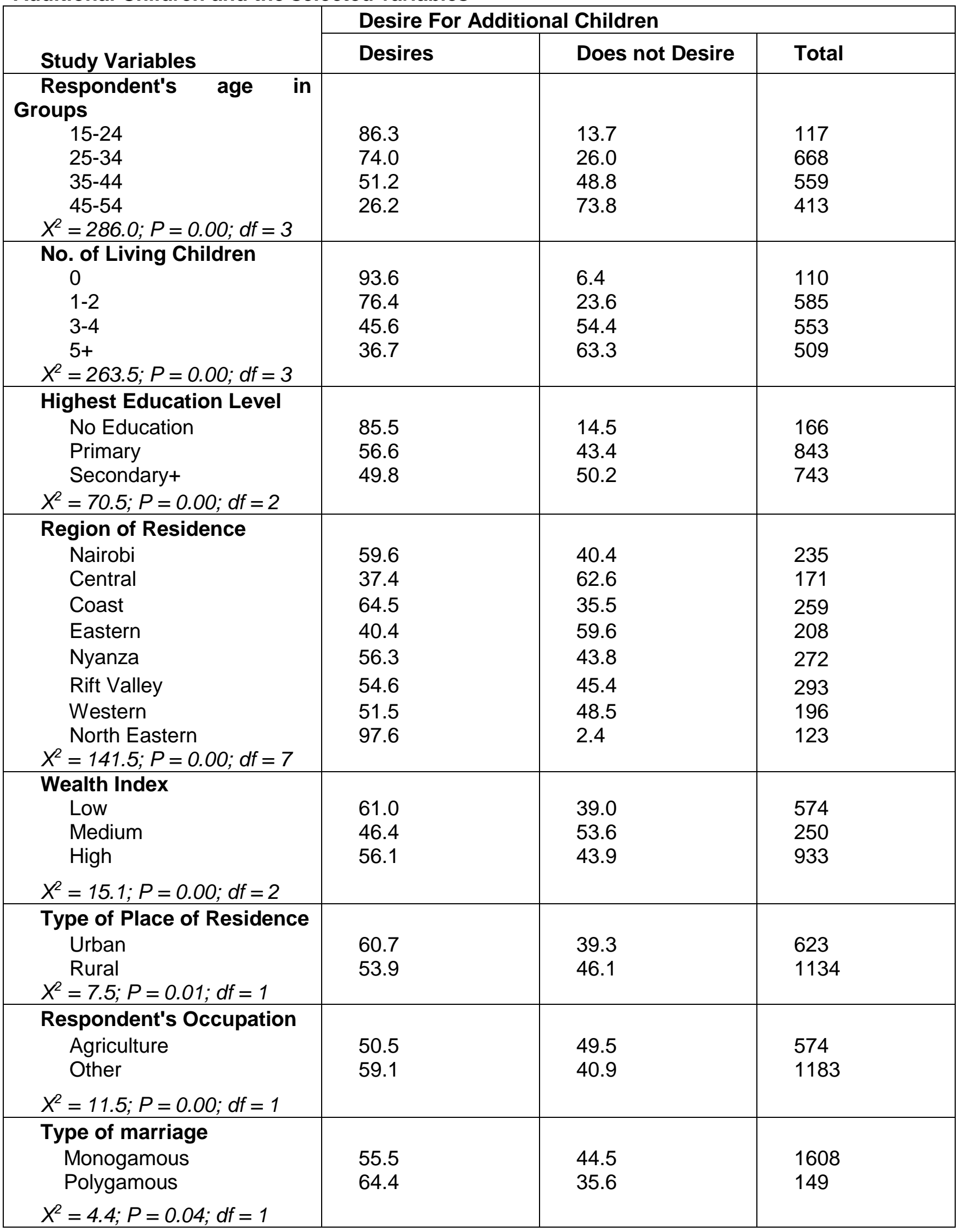


Percentage distribution of the Study Population according to the Desire for Additional Children and the selected variables (Continued)

\begin{tabular}{|l|l|l|l|}
\hline Study Variables & Desires & Does not Desire & Total \\
\hline Total No. of Living Sons & & & \\
0 & 85.0 & 15.0 & 360 \\
$1-2$ & 54.2 & 45.8 & 944 \\
$3-4$ & 36.9 & 63.1 & 333 \\
$5+$ & 40.0 & 60.0 & 120 \\
$X^{2}=185.9 ; P=0.00 ; d f=3$ & & & \\
\hline
\end{tabular}

Note: Level of Significance: ${ }^{\star} \mathrm{P} \leq 0.05 ;{ }^{* \star} \mathrm{P} \leq 0.01 ;{ }^{* \star *} \mathrm{P} \leq 0.001$

It is noted that desire for additional children is inversely associated with age of married men.

Approximately, 86 percent of married men who are below 25 years, desire to have additional children while about 27 percent of the respondents aged between 45-54 years desire to have additional children. The relationship between the two variables is statistically significant at the 0.00 level of significance.

The results clearly show that there is a significant association between desire for additional children and the number of living children. The association between the two variables is statistically significant at the 0.00 level of significance. The findings further illustrate that the association is inversely related and as expected, the more the number of living children one has, the lower the desire for additional children. The findings are also in line with the findings of other studies like Muganzi and Takona (1994). Although they were interested with the fertility preference of women, this study confirms that the fertility preference of men in Kenya is significantly associated with the number of living children a man already has.

The findings of the study showed that respondent's education level is an important determinant of fertility preference. Education has a negative effect on the respondent's desire for additional child. Approximately, 86 percent of married men with no education desire additional children. This is nearly twice the proportion of the married men with higher education who desire additional children. Generally, about 56 percent of men with primary education and about 50 percent of those with secondary + education desire additional children.

The association between education and desire for additional children is highly significant at 0.00 level of significance. This findings point out the fact that, educated men prefer small family sizes. Some of the reasons alluded to this is because educated men appreciate the financial implication of supporting large families, marry at a later age and are more likely to approve family planning as put forward by

http://aps.journals.ac.za previous studies (Uche and Isiugo, 1994; DeRose et al. 2002; Ezeh et al.,1993)

There is an association between men's desire for additional children and their region of residence. The results reveal that almost all married men (98 percent) residing in North Eastern region desire additional children whereas, less than half of married men living in Central and Eastern provinces desire additional children $(37.4 \%$ and $40.4 \%$ respectively). The rest of the regions seem to have almost same proportions who desire additional children ranging from 52 percent in Western and 64 percent in Coast.

Regarding the type of place of residence, it was expected that a larger proportion of men residing in the rural areas would desire to have additional children compared to their urban counterparts, but the opposite is true. Approximately, 61 percent of married men residing in the urban and 54 percent residing in the rural areas desire additional children. In addition the results contrast with the findings from other related studies like Ekisa and Hinde (2006). The association between the two variables is statistically significant at 0.01 level of significance.

A cross tabulation between men's desire for additional children by their wealth index reveals that there is a significant relationship between the two variables. From the findings of this study, about 61 percent of married men from the low wealth index, 46 percent from the medium wealth index and about 56 percent from the high wealth index desire additional children. Results of the chi-square analysis reveal that wealth index and desire for additional children are highly significant at 0.00 level of significance.

Concerning occupation, about 51 percent of married men whose occupation is agriculture desires additional children compare to 59 percent whose occupation is non-agriculture. It was expected that, married men whose occupation is non-agriculture are less likely to desire additional children compared to those in agriculture but the findings refutes it. This could 
be attributed to the limitation of the study in regard to the unequal distribution of married men in different categories of occupation. The results also contrast with the findings of other previous studies (Bankole, 1995), however they are consistent with the findings of Ali (2000) and Rokhsana (2001). The association between occupation and desire for additional children is highly statistically significant at 0.00 significance level.

The number of living sons that a married man has is significantly associated with the desire for additional children. The proportion of married men desiring more children was highest (85 percent) for those who reported having no living son. The desire for additional children reduces as the number of living sons increase. The findings are in conformity with other findings that there is preference for sons in most subSaharan African countries for economic and cultural reasons (Dahl and Moretti, 2008). The findings indicate that the type of marriage has a significant effect on the desire for additional children. It was found that polygamous men compared to monogamous men are more likely to desire additional children. About 65 percent polygamous men desire additional children compared to 56 percent monogamous men. The finding conforms with the hypothesis that 'monogamous men are likely to have lower fertility preference compared to polygamous men'. In addition, the results are consistent with related studies previously done (Ampofo 2000; Wakaranja, 1987; De Rose et al., 2002).

\section{Results of Multivariate Analysis}

This section presents the results of logistic regression. Multivariate analysis involved the use of binary logistic regression to establish the factors that have a significant influence on the desire for additional children. This method of analysis was used so as to examine the net effects of the demographic, socio-economic and socio-cultural factors on the respondents' desire for additional children. Logistic regression analysis was done at three levels and the results presented in three models as shown in table 7.31.

\section{Discussion on Correlates of the desire for additional children}

As illustrated in Table 7.31, out of the five socioeconomic variables introduced into the regression equation in Model I, two variables failed to be statistically significant with the desire for additional children (wealth index and place of residence). The three variables that were found to be significant with the desire for additional children include education, occupation and region, however the level of significance vary. For instance, with regards to education, a married man with primary education is 0.60 times less likely to desire additional children compared to those with no education. The results further reveal that a married man with secondary+ education selected at random will be 0.75 times less likely to desire additional children respectively compared to a married man with no education which was the reference category.

This finding can be explained by the fact that educated men tend to have smaller families because they appreciate the financial implication of supporting large families. In general, men who are educated tend to marry at a later age and tend to adopt the western culture which favors a small family size. Other studies that have conducted in line with this study (Ali, 2000; Rokhsana, 2001; Ayehu, 1998) confirm that education has a significant effect in the individual's fertility-related behaviour. The association between education (primary and secondary + ) and the desire for additional children is highly significant at 0.00 level of significance. Hence the hypothesized association of desire for additional children with education is confirmed.

Occupation was found to be a significant predictor on the desire for additional children at 0.05 level of significance. From the results, married men who are working in the occupation other than agriculture are 0.21 times less likely to desire additional children compared to those involved in agriculture work. According to previous studies, fertility preference of men of different occupational status vary due to the difference of knowledge and educational level and because of the fact that people in the agriculture may value children as a source of labour and also as 'old age support' (Ali, 2000; Rokhsana, 2001). Thus the results confirm the hypothesis that men whose occupation is not in the category of agriculture are more likely to have lower fertility preference. 
Table 7.31: Logistic regression coefficients of the factors influencing the Desire for Additional Children

\begin{tabular}{|c|c|c|c|c|}
\hline & & Model I & Model II & Model III \\
\hline Study Variables & & $\begin{array}{l}\text { Odds } \\
\text { Ratio }\end{array}$ & $\begin{array}{l}\text { Odds } \\
\text { Ratio }\end{array}$ & Odds Ratio \\
\hline & No Education & 1.000 & 1.000 & 1.000 \\
\hline Education & Primary & $0.403^{\star * *}$ & $0.196^{* \star *}$ & $0.214^{\star * *}$ \\
\hline & Secondary + & $0.249^{* * \star}$ & $0.102^{* \star *}$ & $0.113^{* \star \star}$ \\
\hline & Low (ref) & 1.000 & 1.000 & 1.000 \\
\hline Wealth Index & Medium & 0.945 & 1.109 & 1.120 \\
\hline & High & 1.086 & 0.835 & 0.825 \\
\hline & Agriculture (ref) & 1.000 & 1.000 & 1.000 \\
\hline uccupation & Non Agriculture & $0.789^{*}$ & 0.935 & 0.968 \\
\hline Place of Recidenco & Urban (ref) & 1.000 & 1.000 & 1.000 \\
\hline rlace ol hesidence & Rural & 0.869 & 0.930 & 0.915 \\
\hline & Nairobi (ref) & 1.000 & 1.000 & 1.000 \\
\hline & Central & $0.429^{\star * \star}$ & $0.397^{* * *}$ & $0.379^{\star \star \star *}$ \\
\hline & Coast & 1.146 & 1.560 & 1.554 \\
\hline Reginn & Eastern & $0.466^{* * *}$ & $0.523^{*}$ & $0.51^{*}$ \\
\hline negion & Nyanza & 0.964 & 1.178 & 1.039 \\
\hline & Rift Valley & 0.810 & 0.885 & 0.857 \\
\hline & Western & 0.765 & 0.956 & 0.934 \\
\hline & North Eastern & $16.459^{\star \star \star}$ & $30.307^{\star \star \star}$ & $30.106^{\star * *}$ \\
\hline & $45-54$ (ref) & & 1.000 & 1.000 \\
\hline & $15-24$ & & $5.761^{* * *}$ & $5.943^{\star \star *}$ \\
\hline Respondent's Age & $25-34$ & & $5.463^{* * *}$ & $5.327^{\star \star \star \star}$ \\
\hline & $35-44$ & & $3.086^{\star * \star}$ & $3.162^{\star \star \star \star}$ \\
\hline & 0 (ref) & & 1.000 & 1.000 \\
\hline No. of & $1-2$ & & $0.234^{\star \star *}$ & $0.421^{*}$ \\
\hline Children & $3-4$ & & $0.061^{* \star \star}$ & $0.132^{\star \star \star}$ \\
\hline & $5+$ & & $0.038^{\star \star \star}$ & $0.082^{\star \star \star}$ \\
\hline Type of Marriage & union(1) & & & $2.743^{\star \star \star}$ \\
\hline & 0 & & & 1.000 \\
\hline No of I iving Sonc & $1-2$ & & & $0.433^{\star \star \star}$ \\
\hline NNO. OI Living soms & $3-4$ & & & $0.361^{* \star \star}$ \\
\hline & $5+$ & & & $0.314^{\star * *}$ \\
\hline
\end{tabular}

Note: Level of Significance: ${ }^{*} \mathrm{P} \leq 0.05 ;{ }^{* \star} \mathrm{P} \leq 0.01 ;{ }^{* \star *} \mathrm{P} \leq 0.001$

Further, the results showed that region was a significant factor that influenced the desire for additional children. Out of all the regions introduced on the regression equation, Central, Eastern and North Eastern were found to be highly significant at 0.00 significance level. This means, a probability that a married man living in Central and Eastern selected at random would desire additional children is about 0.57 and 0.53 times less likely to desire additional children respectively compared to a married man living in http://aps.journals.ac.za
Nairobi which was the reference category. Married men from North Eastern were 16.459 times more likely to desire additional children compared to married men from Nairobi. The findings are consistent with previous studies done on fertility. Ekisa and Hinde (2006) found that fertility levels were higher in Coast and Western provinces while Kimondo (2003) discovered that, the highest proportion of married men desiring additional children resides in Coast province. However it is important to 
note that Kimondo was doing a comparative study between Coast and Central but not a study involving all the regions.

In Model II, the demographic variables (respondent's age and the number of living children) in addition to the socio-economic variables were introduced into the regression equation. The results showed that respondent's age was a significant predictor of the desire for additional children and the dummy variables 1524 ; 25-34 and 35-44 age groups were found to be highly significant at 0.00 level of significance. Generally, the desire for additional children decreases as age increases. For instance, married men in the age groups $15-24 ; 25-34$ and 35-44 were $5.761 ; 5.463$ and 3.086 times more likely to desire additional children respectively compared to those in the 45-54 age-group which is the reference category.

The number of living children was discovered to be a significant predictor of men's fertility preference in Kenya. As expected, the desire for additional children fall as parity rises. This is because as parity rises, couples meet their desired family sizes. The results illustrated that the fewer the number of living children a man has, the more the likelihood that he will desire additional children. Married men with 1-2; 3-4 and 5 or more living children are $0.77,0.94$ and 0.96 times less likely to desire additional children respectively compared to married men with no living children. From this finding, it is clearly illustrated that the desire for additional children decreases as the number of living children increase. Like age, all the dummy variables designating number of living children are highly significant at 0.00 level of significance. The results are as expected and are in conformity with the descriptive analysis of this study and other studies (Muganzi and Takona, 1994; Kimondo 2003)

In this model, the results also revealed that the introduction of the demographic variables into the regression equation, does not markedly change the nature in which the socio-economic (Education, Region) variables affect the desire for additional children. For instance, with respect to education, married men with primary and secondary + are 0.80 and 0.90 times less likely to desire additional children respectively compared to married men with no education. Similar to the effect of education on Model I, the dummy variables designating education remain highly significant at 0.00 level of significance. With regards to region, married men from Central and Eastern are 0.60 and 0.48 times less likely to desire additional children compared to married men from Nairobi. While married men from North Eastern are 30.307 times more likely to desire additional children compared to married men from Nairobi. In this model, the level of significance of Eastern is reduced to 0.05 significance level. Unlike Model I, the effect of occupation in Mode II ceases to be significant.

In the final model (Model III), the sociocultural variables were introduced into the logistic regression equation. In this model, education, region, age and number of living children still remain significant predictors of the desire for additional children.

The findings confirm that education has a significant influence on fertility preference of currently married men in Kenya. It revealed that increase in education decreases a man's desire for additional children. It is evident that even a few years of schooling (primary level education) creates a highly significant difference on married men's fertility preference. Education can influence men's fertility preference by changing perspectives and lifestyles that are consistent with lower fertility and higher 'quality of children', encouraging partner communication and favorable attitudes towards contraceptive use and health care seeking behaviour. Younger married men are found to be more likey to desire additional children compared to older ones. This illustrates an inverse relationship between respondents age and desire for additional children. This could be attributed to the fact that, i) as the parent grow, they will have achieved their desired family size; ii) the changing reproductive norms where the younger men who are likely to be more educated may be motivated more easily to accept modern ideas and act as fore runners of the new reproductive norms; iii)the foundations of marriage being companionship and procreation; combined with societal pressure the later carries more force among the younger couples compared to older couples.

Because of the inherently differing background factors in various regions, there is an extreme disparity of the proportion and magnitude as well as the direction of men desiring to have additional children. Out of the eight regions, three (Central, Eastern and NorthEastern) were found to be significantly associated with the desire for additional children at both bivariate and multivariate levels of analysis. If a random selection was to be done, a married man in North-Eastern region is 30.106 times more likely to desire additional children compared to a man living in Nairobi. High fertility preference of married men in North Eastern could be attributed to the fact that almost half of the married men sampled in the North Eastern 
had no education compared to other regions. It is also important to note that, compared with the other provinces, fewer households and clusters were surveyed in North Eastern province because of its sparse population, however a deliberate attempt was made to oversample urban areas to get enough cases for analysis.

The study revealed a significant effect of type of marriage and desire for additional children at 0.00 level of significance. According to the findings of this study, polygamous men are 2.743 times more likely to desire additional children compared to monogamous men. This reveals the men's ability to implement their fertility preferences across several unions so as to achieve their desired family size especially with regards to sex preference. Other studies have also shown that men use the avenues of polygamy to achieve their fertility desires in cases where there are disputes within the marriage. Therefore men are able to preserve their reproductive autonomy of wanting more children even during the course of general fertility decline. Thus the hypothesized association of the desire for additional children with type of marriage is confirmed. This finding is also in conformity with other studies done in the developing countries (Ezeh et al., 1996; Blanc et al., 1996; WaKaranja 1987).

Finally, the results established a significant relationship between number of living sons that a man has and his desire for additional children. It was discovered that, a married man with 1-2 living sons is 0.57 times less likely to desire additional children compared to one with no living sons while a man who has 3-4 living sons is 0.64 times less likely to desire additional children compared to one who has no living son. The study further revealed that, a married man, having at least 5 living sons is 0.69 times less likely to desire additional children compared to a man with no living children. The general trend presented by this finding is that childbearing is structured largey around a targeted number of sons rather than family size. This could be explained on the basis of sons perpetuating family name; the need for sons to exercise power in violent areas or to assure household security. In addition, the inheritance laws that rendered sons crucial to retain the family property could be a reason for son preference. The association between the two variables is highly significant at 0.00 significance level. This finding is supported by literature from other studies that have revealed sons being preferred in the sub-Saharan African countries particularly for economic and cultural reasons (Kimondo 2003; khan and Sirageldin, 1977). It also conforms to the results from the descriptive analysis and as hypothesized; sex preference has an effect on the fertility preference.

\section{Conclusion}

Fertility preference in this study was measured in terms of "desire for additional children". This measure can indicate future reproductive behaviour provided that the required family planning services are available, affordable, and accessible to allow people to realize their fertility preferences. Other measures in the fertility decision making process like ideal family size, how soon to have another child, etc. were not considered. Given the likelihood that fertility preference is not static (that is what you prefer today may not be the same in the near future), this study made an assumption the desire for the married men to have an additional child as stated during the interview, remains fixed.

The study had a number of limitations. One, given that Demographic and Health Surveys employ a sample population, biases like age misreporting and response errors are likely to affect the findings of the study. However data quality checks were conducted to ensure that the data collected was appropriate for use.Two, attitudinal variables that are important in analyzing the situation related to fertility preference such as value of children and attitude towards women's status could not be analyzed since the study employed secondary data which was more quantitative than qualitative. Three, the study is specific on the men's fertility preference despite the fact that fertility decisionmaking is a process involving a couple. This is justified since the study also focuses on the determinants of fertility preferences of married men leaving out the single men and as previously stated, husbands play a greater role as far as decision making is concerned on all issues including fertility intentions.

The study has revealed that fertility preference of currently married men in Kenya is influenced by demographic, socio-economic and socio-cultural factors. From the bivariate analysis, all variables which were selected and hypothesized turned out to be significant. However, at the multivariate analysis, the most significant factors influencing a man's desire for additional children include age; number of living children; education; region; type of marriage and number of living sons.

\section{Recommendations}

This study recommends that education for men should be emphasized because education was discovered to have a significant negative effect 
on the fertility preference. Policies that aim at integrating population into development should be encouraged so as to foster socio-economic development in all the regions and hence minimize the regional disparities as it relates to fertility preferences.

In terms of research, further studies, both qualitative and quantitative, to be carried out in order to explore the socio-cultural and religious beliefs, norms and attitudes of men in regards to the value of children.

A detailed qualitative study needs to be conducted specific to the North Eastern region to find out the driving forces for glaringly high fertility preference other than low literacy level.

\section{References}

Ali, M. (2000). The effect of selected sociodemographic characteristics on desire of additional children among couple's in Bangladesh. Master's thesis, faculty of graduate studies, Mahidol University, Thailand.

Ayehu, T.Y. 1998. Correlates of fertility preference of Kenyan women: Evidence from Kenya Demographic and Health Survey.Unpublished Msc thesis. University of Nairobi

Ampofo, A. 2000.Gender inequalities, power in unions, and reproductive decision making in Ghana.Ph.D. diss, Vanderbilt University, Nashville, TN.

Adebayo, A. 1985. "Male Attitudes: Nigeria's Population Predica-ment." Third World International

Bankole A., 1995. Desired fertility and fertility behavior among the Yoruba of Nigeria: A study of couples' preferences and subsequent fertility. Population Studies

Bankole, A., Singh, S., 1998.Couples' Fertility and Contraceptive Decision-Making in Developing Countries: Hearing the Man's Voice. International Family Planning Perspectives 24(1).

Becker, G.S. 1960. An economic analysis of fertility, demographic and economic change in developing countries. University's national bureau committee for economic research.

Blanc, A. K.; Wolff, B.; Gage, A.; Ezeh, A. C.; Neema, S.; Ssekamatte-Ssebuliba, J., 1996. Negotiating reproductive outcomes in Uganda. Calverton, MD, and Kampala, Uganda: Macro International Inc. and Institute of Statistics and Applied Economics.

Bulatao, R.A. and Fawcet, J.T. 1983. Influence on childbearing intentions across the fertility career; demographic and social economic factors and the value of children. East West Centre, Honolulu.

Caldwell, J.C. (1976). "Toward a restatement of demographic transition theory". Population and Development Review (Population Council) 2 (3/4): 321-366

Caldwell, J.C. 1982. Theory of Fertility Decline. London: Academic Press.

Dahl, G., Moretti, E., 2008. The Demand for Sons. Review of Economic Studies

DeRose, F. L., Dodoo, F. N. and Patil V., 2002. Fertility Desires and Perceptions of Power in Reproductive Conflict in Ghana. Sage Publications, Inc.

Ekisa, Hinde \& Andrew., 2006. Fertility Transition in Kenya: A Regional Analysis of Proximate Determinants. Southampton, UK, Southampton Statistical Sciences Research Institute.

Easterline, R.A. 1973. An economic framework for fertility analysis. Studies in family planning, 6(3).

Ezeh, A.C., 1993. The influence of spouses over each other's contraceptive attitudes in Ghana.Studies in Family Planning.May-June

Ezeh, A.C. et al., 2005. Men's Influence on the Onset and Progress of Fertility Decline in Ghana 1988-98, Population Investigation Committee

Foreit, K.G., and M.H. Suh. 1980. The effect of reproductive intentions on subsequent fertility among low parity Korean women 1971-1976. Studies in Family Planning 11(3): 91-104.

Isiugo-Abanihe, U.C., 1994.Reproductive motivation and family size preferences among Nigerian men. Studies in Family Planning

Kenya National Bureau of Statistics (KNBS) and ICF Macro. 2010. Kenya Demographic and Health Survey 2008-09. Calverton, Maryland: KNBS and ICF Macro.

Khan, A.M. and I. Sirageldin, 1977. Son preference and demand for additional children in Pakistan. Demography 14(4): 481495.

Kimondo, M., 2003. Determinants of Fertility Preference in Kenya: Unpublished Thesis, University of Nairobi

Lightbourne, R.E. and A.L. McDonald, 1982.Family size preferences.WFS comparative studies, 14 . International statistical institute, Voorburg. Netherlands.

Mahmud, N., and Ringheim, K, (1997). Knowledge, approval and communication about family planning as correlates of desired family among spouses in Pakistan.International Family Planning Perspective, 23:122-129and 145 
Muganzi, Z and Takona, T (1994) Fertility decline and demand for family planning in Kenya. DHS, Macrolnternational Inc. Columbia, Maryland USA.

Mutetei K., 1998. The Proximate Determinants of Fertility: Evidence from the 1999 Kenya Demographic and Health Survey, Unpublished Thesis, University of Nairobi.

Pullum, T. W. 1980. Illustrative Analysis; fertility preferences in Sri-Lanka. A World Fertility Survey Scientific Report. International Statistical Institute.Voorsburg. Netherlands.

Rokhsana, R. (2001). Factors influencing fertility preference of men in Bangladesh population and reproductive health research faculty of graduate studies Mahidol University.

Singh, S., and Casterline, J. (1985). "The Socioeconomic determinants of fertility", In J. Cleland and J. Hobcraft (eds). Reproductive change in developing countries. Oxford University press.199-222.
Udry, J.R. 1983.Do couples make fertility plans one birth at a time? Demography 20(2)

WaKaraja, W. 1987. "Outside wives" and "inside wives" in Nigeria: A study of changing perceptions in marriage. In Transformations of African marriage, edited by D. Parkin and D. Nyamwaya. Man- chester, UK, and Wolfeboro, NH Manchester University Press for the International African Institute

Willis R J., 1973. A new approach to economic theory f fertility, in Shultz, W (ed) New economic approaches to fertility. Proceedings of a conference. June 8-9, 1972. Journal of political economy 81:14-64 